Psychotherapeut 2015 $\cdot 60: 5-5$

DOI 10.1007/s00278-014-1099-4

Online publiziert: 5. Dezember 2014

c) Springer-Verlag Berlin Heidelberg 2014

Carsten Spitzer ${ }^{1}$. Harald J. Freyberger ${ }^{2}$

${ }^{1}$ Asklepios Fachklinikum Tiefenbrunn, Rosdorf bei Göttingen

${ }^{2}$ Klinik und Poliklinik für Psychiatrie und Psychotherapie der Universitätsmedizin Greifswald, HELIOS Hanseklinikum Stralsund

\title{
Selbstbeschädigung und Selbstverletzung in der Psychotherapie
}

zu teilweise kulturell akzeptierten Aneignungsformen geworden, für die neue (gesellschaftliche und) therapeutische Antworten gefunden werden müssen (Libal u. Plener 2009; Plener 2014).

In diesem Heft der Zeitschrift Psychotherapeut haben wir versucht, diese sehr unterschiedlichen Aspekte aufzugreifen. Christian Schmahl (Mannheim) und Christian Stiglmayr (Berlin) fassen die kognitiv-behavioralen Konzepte zu dieser Thematik zusammen. Dem stellen Ulrich Sachsse und Willy Herbold (beide Göttingen) die psychodynamische Sichtweise gegenüber. Andreas Hill, Wolfgang Berner und Peer Briken (alle Hamburg) haben sich mit dem sog. riskanten Sexualverhalten beschäftigt, das sie zwischen den Polen der Suche nach Lust und der sexuellen Selbstbeschädigung diskutieren. Annegret Eckhardt-Henn (Stuttgart) fasst den gegenwärtigen Kenntnisstand zu den artifiziellen Störungen zusammen und greift dabei ebenso wie Hill et al. die dimensionalen Aspekte auf.

Zwei im engeren Sinn empirische Arbeiten komplettieren dieses Schwerpunktheft. Mestel et al. (Bad Grönenbach) fassen Daten zu Prävalenz und therapeutischer Beeinflussbarkeit selbstverletzenden Verhaltens bei Patienten mit strukturellen Störungen im Bereich der psychosomatischen Rehabilitation zusammen. Sie zeigen, dass die Häufigkeit sich selbst verletzender Patienten in der psychosomatischen Rehabilitation in ähnlichen Größenordnungen einzuschätzen ist wie in vergleichbaren Versorgungssegmenten und die symptomspezifischen Effektstär- ken, die durch die Behandlung erreicht werden, eher als begrenzt aufgefasst werden müssen. Spitzer et al. (Tiefenbrunn) beschäftigen sich mit den differenziellen Effekten von Kindheitstraumatisierungen auf das spätere autoaggressive Verhalten bei Patienten mit Borderline-Persönlichkeitsstörungen. Sie können u. a. zeigen, dass Depression und Dissoziation als bedeutsame Prädiktoren für späteres autoaggressives Verhalten in Erscheinung treten sowie als partieller Mediator des $\mathrm{Zu}$ sammenhangs zwischen Missbrauch und Autoaggression aufzufassen sind.

\section{Korrespondenzadresse}

\section{Prof. Dr. Carsten Spitzer}

Asklepios Fachklinikum Tiefenbrunn

37124 Rosdorf bei Göttingen

c.spitzer@asklepios.com

Interessenkonflikt. Carsten Spitzer und Harald J. Freyberger geben an, dass kein Interessenkonflikt besteht.

\section{Literatur}

Clarkin JF Yeomanns FE (2008) Psychotherapie der Borderline-Persönlichkeit. Manual zur Psychodynamischen Psychotherapie. Schattauer, Stuttgart Kernberg OF (1978) Borderline-Störungen und pathologischer Narzissmus. Suhrkamp, Frankfurt a. M.

Libal G, Plener P (2009) Selbstverletzendes Verhalten. In: Fegert J, Streeck-Fischer A, Freyberger HJ (Hrsg) Adoleszenzpsychiatrie. Schattauer, Stuttgart, S 190-200

Linehan MM (1993) Cognitive-behavioral treatment of borderline personality disorder. Guilford, New York Plener P (2014) Suizidales Verhalten und nichtsuizidale Selbstverletzungen. Springer, Berlin Heidelberg New York Tokio
Jugendkultur aus dem Schatten der Verheimlichung herausentwickelt und sind 